\title{
Spectral Clustering Based on Multi-scale Stochastic Tree Image Segmentation Algorithm
}

\author{
Yuantao Chen \\ School of Computer and Communication Engineering \\ Changsha University of Science and Technology \\ Changsha, China \\ e-mail: yufeng8552@qq.com
}

\author{
Jingwen Zuo \\ College of ChengNan \\ Changsha University of Science and Technology \\ Changsha, China \\ e-mail: zojw@qq.com
}

\begin{abstract}
For spectral clustering is applied to image segmentation is difficult to calculate the spectral weight matrix of the actual problem, we have defined the pixel distance between the point and the class is given a sampling theorem, the design of a hierarchical image segmentation algorithm in the use of this algorithm for image segmentation. By adjusting the scaling factor to merge or split a large class of smaller classes, so the image segmentation both randomness but also has multi-scale feature, called spectral clustering based on multi-scale stochastic tree image segmentation (SCMSTIS). The experimental results show that the algorithm is effective.
\end{abstract}

Keywords-spectral clustering; graph, multi-scale stochastic tree, image segmentation, SCMSTIS

\section{INTRODUCTION}

Image segmentation is the image segmentation with different characteristics into disjoint connected sub-regions in the process, the results of image analysis and image understandings are important. Clustering is a common image segmentation techniques have been proposed over the years many based clustering image segmentation algorithm, such as K-means [1-2], Fuzzy C-Means [3], EM (Expectationmaximization Algorithm) [4-5], Self-organizing map [6], etc. However, these methods often do not consider the spatial information of an image, resulting in divided areas is not continuous, and most cases can't get global optimal solution.

The reference [8] had proposed spectral clustering method proposed in this paper through the pixel sampling, and sampling points using the spectral clustering, the remaining tap nearest to solve clustering principle spectral clustering is applied to image segmentation right eigenvalues and eigenvectors of the problem is difficult to calculate this end, this paper defines the pixel distance between the point and the class, followed by a sampling of questions to establish a number of samples with class the proportion of the overall relationship between the final adoption of the pixels between the right edge of the introduction of the scale factor, the design of a recursive hierarchical multi-scale segmentation algorithm, called spectral clustering based image segmentation multi-scale stochastic tree algorithm, to solve the spectral clustering is applied to image segmentation problem is difficult to calculate spectral.

\section{RELATED DEFINITIONS}

Let $I$ is a picture image of $m \times l$, denoted $N=m \times l$, and $N \geq 10^{4} . V=\left\{v_{1}, v_{2}, v_{3}, \ldots, v_{N}\right\}$ is the set of pixels, where $v_{i}$ is the $\mathrm{i}$-th pixel. construct the set of vertices in $V$ weighted graph $G(V, E, W)$, where $E=V \times V$ represents the set of edges between vertices, for convenience with $e_{i j}$ between vertices $v_{i}$ and $v_{j}$ edges $\left(v_{i}, v_{j}\right)$, i.e. $e_{i j}=\left(v_{i}, v_{j}\right), W$ is an element of the right to the right side of the matrix. $w_{i j}$ is located on the edge of the right edge $e_{i j}$, then $w_{i j} \in[0,1]$ represents between $v_{i}$ and $v_{j}$ the degree of similarity, $v_{i}$ and $v_{j}$ more similar between that $v_{i}$ and $v_{j}$ are more likely to belong to the same class, $w_{i j}$ greater; otherwise, $w_{i j}$ greater, indicating that $v_{i}$ and $v_{j}$ are more likely to belong to the same class, especially $w_{i i}=1$, $i=1,2, \ldots, N$.

Using the above notation, the image segmentation is in accordance with certain rules set $V$ into disjoint subsets and, namely:

$$
\begin{aligned}
& V=\bigcup_{i=1}^{k} V_{i}, V_{i} \cap V_{j}=\varnothing, i \neq j, \\
& i, j=1,2, \ldots, k
\end{aligned}
$$

Where $k$ is the number of categories, $V_{i}$ is the $\mathrm{i}$-th divided category, $i=1,2, \ldots, k$.

Definition 1 (The distance between two pixels): It is defined as the binary function of $d: V \times V \rightarrow R^{+}$, if the binary function $d$ satisfies:

(1) $\forall u, v \in V, d(u, v)=d(v, u)$.

(2) $\forall u, v \in V, u$ and $v$ are more similar, $d(u, v)$ is smaller, especially when the $u$ and $v$ coincide, 
$d(u, v)=0, d$ is called the two pixels the distance between the function.

Definition 2 (Two similarity of two pixels): define the binary function of $R: V \times V \rightarrow[0,1]$, if the binary function R satisfies:

(1) $R\left(v_{i}, v_{j}\right)=R\left(v_{j}, v_{i}\right)=w_{i j}, i, j=1,2, \ldots, N$.

(2) There exists a unary function of $f: R^{+} \rightarrow[0,1]$, such that $\forall u, v \in V \quad$ with $R(u, v)=f(d(u, v))=f(d)$, then $R$ is called two similarity function between pixels.

Obviously:

$$
W=\left(w_{i j}\right)_{N \times N}=\left(R\left(v_{i}, v_{j}\right)\right)_{N \times N}=\left(f\left(d\left(v_{i}, v_{j}\right)\right)\right)_{N \times N}
$$

Definition 3 (Distance between the class and pixel) set pixel of $v \in V$, Class $A \subset V$, define the distance between $v$ and $A$.

$$
D(v, A)=1-\max \left\{R\left(v, v_{j}\right) \mid v_{j} \in A\right\}
$$

Let $|A|$ represents set $A$ trend.

\section{SpeCtral Clustering Based On Multi-SCAle STOCHASTIC TREE IMAGE SEGMENTATION}

This section reviews the reference [8] proposed spectral clustering algorithm, and then the image pixels in the study sample is obtained based on a sampling theorem, and finally according to the theorem based on spectral clustering image multi-scale stochastic tree segmentation algorithm.

\section{A. Spectral Clustering Algorithm based on Weight Matrix}

The reference [8] perturbation theory using matrix spectral clustering analyzes, and presents a spectral clustering algorithm based on weight matrix (SCAWM), the algorithm can be described as follows:

Step 1: Clustering according to the given set $V$ of points to be constructed weighted graph of $G(V, E, W)$;

Step 2: Calculating $W$ eigenvalues of $\lambda_{1} \geq \lambda_{2} \geq \ldots \geq \lambda_{N}$ and corresponding unit orthogonal eigenvectors in $x_{1}, x_{2}, \ldots, x_{N}$;

Step 3: $k=\max \left\{i \mid \lambda_{i}>1, i=1,2, \ldots, N\right\}$ calculated by number of categories:

Step 4: Move $W$ largest $k$ eigenvalues corresponding unit eigenvectors orthogonal matrix by columns:

$$
X=\left(x_{1}, x_{2}, \ldots, x_{k}\right)=\left(\alpha_{1}^{T}, \alpha_{2}^{T}, \ldots, \alpha_{n}^{T}\right)^{T}
$$

Step 5: Calculating $\frac{\alpha_{i} \alpha_{j}^{T}}{\left\|\alpha_{i}\right\|_{2}\left\|\alpha_{j}\right\|_{2}}$, and it is classified according to the value of $\frac{\alpha_{i} \alpha_{j}^{T}}{\left\|\alpha_{i}\right\|_{2}\left\|\alpha_{j}\right\|_{2}}$. Selecting the threshold flag value, so that:

$$
R\left(v_{i}, v_{j}\right)=\left\{\begin{array}{l}
1, \frac{\alpha_{i} \alpha_{j}^{T}}{\left\|\alpha_{i}\right\|_{2}\left\|\alpha_{j}\right\|_{2}} \geq \text { flag } \\
0, \frac{\alpha_{i} \alpha_{j}^{T}}{\left\|\alpha_{i}\right\|_{2}\left\|\alpha_{j}\right\|_{2}}<\text { flag }
\end{array}\right.
$$

If $R\left(v_{i}, v_{j}\right)=1 v_{i}$ and $v_{j}$ will be classified as a class. If $R\left(v_{i}, v_{j}\right)=0$, then the $v_{i}$ and $v_{j}$ classified into different classes.

Step 6: The calculated number of classes, if the class number is equal to $k$ then clustering the end, otherwise return to step 5 to re-select parameter flag.

\section{B. Spectral Clustering Based on Multi-scale Stochastic \\ Tree Image Segmentation Algorithm}

According to section B's conclusions drawn from $V$ if 50 samples consisting of $V_{s}$, then there is:

$$
P\left(V_{s} \cap V_{i} \neq \varnothing\right)>0.95, i \in\left\{j \mid \frac{\left|V_{j}\right|}{|V|} \geq 0.1\right\}
$$

So if 50 samples are drawn from $V$ and 50 samples with SCAWM clustering algorithm, then the principle of using the nearest pixel point and the distance between classes to other elements of a cluster can get $\mathrm{V}$ split. noted that according to the sampling theorem, the segmentation method is conducive to segment the class from $\mathrm{V}$ larger proportion of the class, which may result in less image segmentation (It is segmented from the image if the area should continue to be divided, then the images are referred to herein due to segmentation). To solve this problem, we consider the recursive segmentation method for segmentation of the class division continue, since the image will be generated for recursive partitioning a "tree", and because each partition requires random sampling, so get will be a random tree, so this article called random tree image segmentation.

In the above image random tree split in favor of the first division despite a larger proportion of the class of the class, but because of the randomness of the sampling may also produce an image of the over-segmentation in order to solve this problem, we consider the similarity function $R=f(d)$ the introduction of the scale factor to be modulated, so that $R^{*}=f(d / \sigma)$, where $\sigma$ is the scale 
factor, and so that the right side on the edge $e_{i j}$ is $R^{*}\left(v_{i}, v_{j}\right), i, j=1,2, \ldots, N$, then the divided process by dynamically adjusting the value of $\sigma$ to prevent oversegmentation, specific practices are as follows: initial segmentation of the image, take a larger $\sigma$ to merge smaller classes, resulting in an image obtained when compared with the initial segmentation "rough" scale division; subsequent segmentation of the image through the process of gradually reducing $\sigma$ to get the image more "small" partition so that the role of the scale factor, on the one hand to prevent oversegmentation of the image, on the other hand makes the image segmentation from the "coarse" to "fine" in, got a multi-scale image segmentation.

Summing up the above can be obtained as follows based on spectral clustering image segmentation algorithm multiscale stochastic tree:

Step 1: The pixels in the image $I$ constructed weighted graph with vertex set of $G(V, E, W)$;

Step 2: For selected small positive number of $\alpha$, calculate the value of $b$, determine the number of samples $n$;

Step 3: Not back from $V n$ samples taken at random in $V_{s}$, select the appropriate scale factor to modulate $\sigma$ the similarity function $R$, and use SCAWM to cluster $V_{s}$ for the upcoming $V_{s}$ expressed as:

$$
V_{s}=\underset{i=1}{V_{i}^{k}} V_{s}^{(i)}, V_{s}^{(i)} \bigcap V_{s}^{(j)}=\varnothing, i \neq j, i, j=1,2, \ldots, k
$$

Step 4: According to $\forall v \in V-V_{s}$ to calculate $D\left(v, V_{s}^{(i)}\right) \quad, \quad i=1,2, \ldots, k$

$D\left(v, V_{s}^{\left(i_{0}\right)}\right)=\min _{1 \leq i \leq k}\left\{D\left(v, V_{s}^{(i)}\right)\right\} \quad$, then make $V_{s}^{\left(i_{0}\right)}=V_{s}^{\left(i_{0}\right)} \cup\{v\}$. In the $V-V_{s}$ for each element of the clustering process, the division $V$ finally obtained:

$$
V=\bigcup_{i=1}^{k} V_{s}^{(i)}, V_{s}^{(i)} \cap V_{s}^{(j)}=\varnothing, i \neq j, i, j=1,2, \ldots, k
$$

Step 5: Selecting the appropriate scale factor of $\sigma$, for the separation of subclasses. Repeat the above step 1 to step 5 .

In the use of SCMSTIS to image segmentation, there are two parameters need to be determined: the sample size $\mathrm{n}$ and the scale factor of $\sigma$. According to the discussion of section $B$, in the general case, the sample size $n=50$ to solve the problem. Determine the scale factor $\sigma$ is the spectral clustering applications a difficult problem, the reference [7] gives an empirical method for determining the reference [9] proposed using learning methods, depending on the image content to determine the appropriate scale factor, first proposed the use of large-scale factor for coarse image segmentation, and then use small-scale factor of the image to continue dividing both fine complement to the above method, but also is an attempt to determine the scale factor. SCMSTIS can be applied directly interact image segmentation, through the choice of the scale factor can not only control the segmentation process artificially, but also on the region of interest for detailed segmentation, while not interested in the area is not split, which in some applications, such as military target identification and medical diagnosis has a good application value.

\section{EXPRIMENTAL RESULTS AND ANALYSIS}

In order to verify the validity of SCMSTIS, this paper some synthetic and real images on the experiments selected parameters: $\alpha=0.05, b=-1.65$, the number of samples $n=50$.

\section{A. Algorithm Verification}

In order to verify the validity of SCMSTIS, this paper will be applied to the synthetic image segmentation, and then in the sub-synthetic and real images and more experiments on a large number of experimental results show SCMSTIS has a good segmentation performance.

Figure 1 is the SCMSTIS for a color image segmentation results. Selected similarity function is:

$$
R_{2}=f\left(\frac{d_{2}}{\sigma_{2}}\right)=\exp \left(-\frac{d_{2}}{\sigma_{2}}\right)
$$

Where $\quad d_{2}(u, v)=\max (|I(u)-I(v)|)^{2}$, $\forall u, v \in V, I(u)=\left(R_{u}, G_{u}, B_{u}\right)$ is triplet, its three components denote the pixel $u$ at the R, G, B values.

$$
\max (|I(u)-I(v)|)=\max \left(\left|R_{u}-R_{v}\right|,\left|G_{u}-G_{v}\right|,\left|B_{u}-B_{v}\right|\right)
$$

Figure $1(\mathrm{a})$ is the original color image segmentation in the first scale parameter $\sigma_{2}=2$, split the results shown in Figure 1(b) below. The first split the image into two categories, at the second segmentation, select the scale factor $\sigma_{2}=0.5$ on one of a class of segmentation, segmentation results shown in Figure 1(c) below.
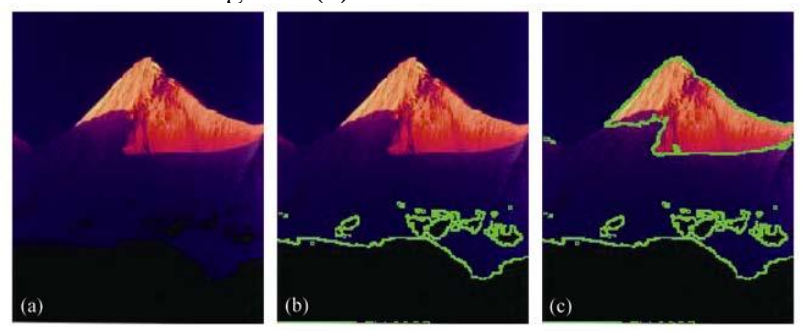

Figure 1. SCMSTIS used in a colorful image segmentation results: (a) Original images $(207 \times 256)$; (b) the first segmentation results of

$$
\sigma_{2}=2 ; \text { (c) the second segmentation results of } \sigma_{2}=0.5
$$




\section{B. Comparative Analysis}

In order to evaluate the performance of the division SCMSTIS, the paper carried out the following two experiments:

With the associated spectral clustering image segmentation algorithm has comparative experiments. The SCMSTIS based Normalized cut [7] and Min-max cut [10] algorithm for image segmentation objective comparison, Figure 2 is a test the results from the experimental results, though they are based on spectral clustering image segmentation algorithm, using SCMSTIS segmentation results significantly better than on Normalized cut and Minmax cut segmentation results. The analysis of the main reason is Normalized cut and Min-max cut during the clustering tend to get two types of similarity within the same class, but he was not SCMSTIS making such a request clustering, so get the class more in line with the actual situation.

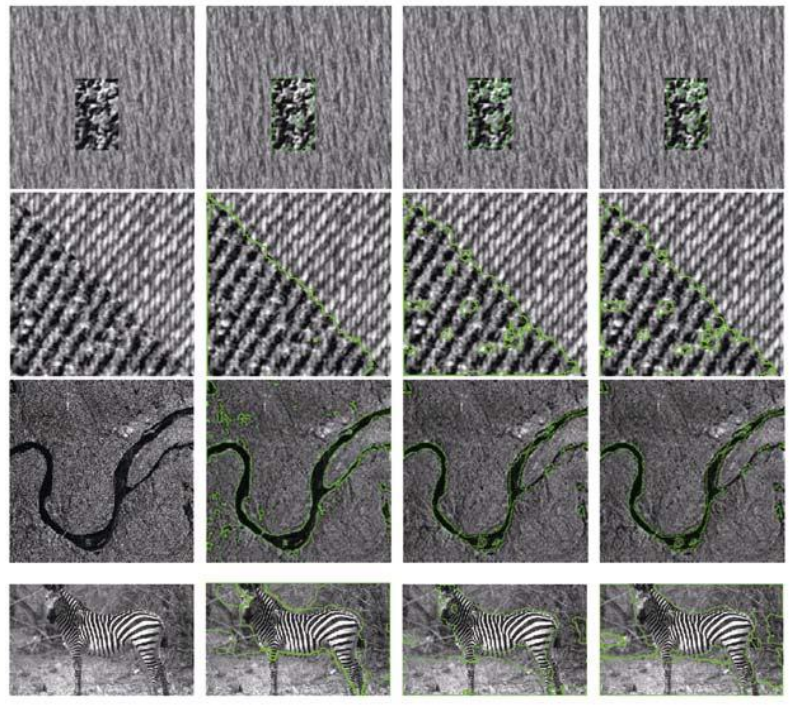

Figure 2. SCMSTIS based Normalized cut and Min-max cut for image segmentation algorithm comparison results: The first column of the original image. The second column is obtained by the use of segmentation results SCMSTIS. The third column is to use Normalized cut segmentation results. The fourth column is obtained using the Min-max cut segmentation results.

\section{CONCLUSIONS}

In this paper, spectral clustering is applied to image segmentation right eigenvalues and eigenvectors is difficult to calculate the actual difficulties presented spectral clustering based on multi-scale stochastic tree image segmentation algorithm (SCMSTIS). This algorithm has the following characteristics: (1) Only a small number of sampling points for the use SCAWM cluster, while the number of non-sampling points using a simple principle for the nearest cluster, thus SCMSTIS can be used for large images (e.g. SAR image) of segmentation; (2) By tree image segmentation to solve the image segmentation problem due to, because every partition has a random, so SCMSTIS is a random tree segmentation method; (3) By introducing a scaling factor so that the image segmentation from "coarse" to "fine" for, as the scale factor varies with the split, so SCMSTIS is a multi-scale segmentation method; (4) It is divided in different scales, in order to get better segmentation results, you can choose a different similarity function, reflects SCMSTIS flexibility.

\section{ACKNOWLEDGMENT}

This work was supported in part by a grant from by the Project Supported by Scientific Research Fund of Hunan Provincial Education Department (No. 12B005), the Hunan Province Undergraduates Innovating Experimentation Project (No. (2013) 191-501) and the Hunan Province Science and Technology Planning (No. 2014SK3080).

\section{REFERENCES}

[1] Kanungo T, Mount D M, Netanyahu N S, et al. An efficient k-means clustering algorithm: Analysis and implementation. IEEE Transactions on Pattern Analysis and Machine Intelligence, 2002, 24(7): 881-892.

[2] Chen C W, Luo J, Parker K J. Image segmentation via adaptive kmean clustering and knowledge-based morphological operations with biomedical applications. IEEE Transactions on Image Processing, 1998, 7(12): 1673-1683.

[3] Shen S, Sandham W, Granat M, et al. MRI fuzzy segmentation of brain tissue using neighborhood attraction with neural-network optimization. IEEE T Inf Technol Biomed, 2005, 9(3): 459-467.

[4] Zhang Y, Brady M, Smith S. Segmentation of brain MR images through a hidden Markov random field model and the expectationmaximization algorithm. IEEE Trans Med Imaging, 2001, 20(1): 4557.

[5] Pal S K, Mitra P. Multispectral image segmentation using the roughset-initialized EM algorithm. IEEE Trans Geosci Remote Sensing, 2002, 40(11): 2495-2501.

[6] Yin H, Allinson N M. Unsupervised segmentation of textured images using a hierarchical neural structure. Electron Lett, 1994, 30(22): 1842-1843.

[7] Shi J, Malik J. Normalized cuts and image segmentation. IEEE Transactions on Pattern Analysis Machine Intelligence, 2000, 22(8): 888-905.

[8] Kannan R, Vempala S, Vetta A. On clustering: good, bad and spectral. Journal of ACM, 2004, 51(3): 597-515.

[9] Bach R, Jordan M I. Learning Spectral Clustering. University of California at Berkeley Technical Report UCB/CSD-03-1249. 2008.

[10] Ding C H Q, He X, Zha H, et al. A min-max cut algorithm for graph partitioning and data clustering. In: Cercone N, Lin T Y, Wu X, eds. ICDM 2009. Los Alamitos, California: IEEE Computer Society, 2009. 107-114. 